\title{
Dietary Exposure to Transgenic Rice Expressing the Spider Silk Protein Fibroin Reduces Blood Glucose Levels in Diabetic Mice: The Potential Role of Insulin Receptor Substrate-1 Phosphorylation in Adipocytes
}

\author{
Ji-Eun Park ${ }^{1}$, Yeon Jae Jeong ${ }^{1}$, Joon Beom Park ${ }^{1}$, Hye Young Kim¹, Young Hyun Yoo', \\ Kwang Sik Lee², Won Tae Yang ${ }^{2}$, Doh Hoon Kim², and ${ }^{\dagger}$ Jong-Min Kim \\ ${ }^{1}$ Dept. of Anatomy and Cell Biology, College of Medicine, Dong-A University, Busan 49201, Korea \\ ${ }^{2}$ College of Life Sciences and Natural Resources, Dong-A University, Busan 49315, Korea
}

\begin{abstract}
Type 2 diabetes mellitus (T2DM) is characterized by insulin resistance (IR). T2DM is correlated with obesity and most T2DM medications have been developed for enhancing insulin sensitivity. Silk protein fibroin (SPF) from spiders has been suggested as an attractive biomaterial for medical purposes. We generated transgenic rice (TR) expressing SPF and fed it to diabetic BKS.Cg-m+/+Lepr ${ }^{\mathrm{db}}$ mice to monitor the changes in blood glucose levels and adipose tissue proteins associated with energy metabolism and insulin signaling. In the present study, the adipocyte size in abdominal fat in TR-SPF-fed mice was remarkably smaller than that of the control. Whereas the adenosine monophosphate-activated protein kinase (AMPK)-activated protein kinase and insulin receptor substrate 1 (IRS1) protein levels were increased in abdominal adipose tissues after TR-SPF feeding, levels of six-transmembrane protein of prostate 2 (STAMP2) proteins decreased. Phosphorylation of AMPK at threonine 172 and IRS1 at serine 307 and tyrosine 632 were both increased in adipose tissues from TR-SPFfed mice. Increased expression and phosphorylation of IRS1 at both serine 307 and tyrosine 632 in adipose tissues indicated that adipocytes obtained from abdominal fat in TR-SPF-fed mice were more susceptible to insulin signaling than that of the control. STAMP2 protein levels decreased in adipose tissues from TR-SPF-fed mice, indicating that STAMP2 proteins were reducing adipocytes that were undergoing lipolysis. Taken together, this study showed that TR-SPF was effective in reducing blood glucose levels in diabetic mice and that concurrent lipolysis in abdominal adipocytes was associated with alterations of AMPK, IRS1, and STAMP2. Increased IRS1 expression and its phosphorylation by TR-SFP were considered to be particularly important in the induction of lipolysis in adipocytes, as well as in reducing blood glucose levels in this animal model.
\end{abstract}

Key words : Spider silk protein fibroin, Transgenic rice, IRS1, Adipocytes, Diabetic mouse

\section{INTRODUCTION}

During the last few decades, the prevalence of diabetes mellitus (DM) has increased worldwide and is one of the leading causes of high mortality and morbidity. DM is a metabolic syndrome characterized by an elevated blood glucose levels and is largely classified into two types: type 1 DM (T1DM) and type 2 DM (T2DM) (Thomas \& Philipson, 2015). T1DM is associated with failure in insulin production resulting from the destruction of pancreatic $\beta$ cells while T2DM often is characterized by insulin resistance (IR). The therapeutic regimes and outcomes for

Manuscript received July 10, 2019, Received in revised form July 31, 2019, Accepted August 13, 2019

${ }^{\dagger}$ Corresponding Author : Jong-Min Kim, Dept. of Anatomy and Cell Biology, College of Medicine, Dong-A University, Busan 49201, Korea. Tel.: +8251-240-2792, Fax: +82-51-245-3872, E-mail: jmkim7@ dau.ac.kr

This is an Open Access article distributed under the terms of the Creative Commons Attribution Non-Commercial License (http:// creative-commons.org/licenses/by-nc/3.0) which permits unrestricted non-commercial use, distribution, and reproduction in any medium, provided the original work is properly cited. 
T2DM is more complicated and unguaranteed, respectively, than those of T1DM. T2DM is often correlated with obesity (Boles et al., 2017) and most T2DM medications have been developed for enhancing insulin sensitivity. For instance, Metformin is the most representative antidiabetic drug, especially for obese individuals (Sanchez-Rangel \& Inzucchi, 2017). Metformin functions by activating one of the enzymes involved in expressing gluconeogenic genes, known as 5-adenosine monophosphate-activated protein kinase (AMPK) (Rena et al., 2017).

Silk protein fibroin (SPF) from spiders has been suggested as an attractive biomaterial for industrial and medical purposes because of its unique properties (Altman et al., 2003; Kluge et al., 2008). SPF is mainly composed of glycine and alanine. The biological effects of SPF include the enhancement of glucose (Hyun et al., 2004; Do et al., 2012) and lipid metabolism (Jung et al., 2010), anti-viral activity (Gotoh et al., 2000), DNA damage protection (Park et al., 2002), and blood pressure-depressing activity (Igarashi et al., 2006). Recombinant spider SPF exhibits non-cytotoxic and non-inflammatory effects in NIH 3T3 cells (Lee et al., 2016) and also reduces blood glucose levels in diabetic mice (Lee et al., 2014).

In the present study, we generated transgenic rice (TR) expressing SPF and fed it to diabetic BKS.Cg-m+/+Lepr ${ }^{\mathrm{db}}$ mice to monitor the changes in blood glucose levels and adipose tissue proteins associated with energy metabolism and insulin signaling. The purpose of this study was to investigate whether cellular and biochemical alterations in adipocytes were correlated with changes in blood glucose levels in TR-SPF-fed mice.

\section{MATERIALS AND METHODS}

\section{Reagents and antibodies}

Bouin's solution, eosin and hematoxylin were purchased from Sigma Chemical Co. (St. Louis, MO, USA). Complete Protease Inhibitor Cocktail Tablet was from Roche Applied
Science (Mannheim, Germany). The western enhanced chemiluminescence (ECL) detection reagent was purchased from Bio-Rad (Hercules, CA, USA). VECTASTAIN ${ }^{\circledR}$ Elite ABC Kit was from Vector Laboratories (Burlingame, CA, USA). Anti-AMPK $\alpha$ and Phospo-AMPK $\alpha(T h r 172)$ antibodies were purchased from Cell Signaling Technology (Danvers, MA, USA). Anti-insulin receptor substrate (IRS1), Phospo-IRS-1 (Ser307) and Phospo-IRS-1 (Tyr632) were obtained from Santa Cruz Biotechnology (Santa Cruz, CA, USA). Anti-STEAP4 (STAMP2) antibody was from Proteintech (Rosemont, IL, USA). Anti- $\beta$-Tubulin antibody was from Sigma Chemical Co. (St. Louis, MO, USA).

\section{Production of transgenic rice}

cDNAs encoding a partial repetitive region and the Cterminal domain of $A v M a S p$ (Lee et al., 2012) were PCRamplified from $p G E M T-A v M a S p$ using the forward primer 5'-GGATCCATGGCCGCCGCAGCCGCA-3' (with BamHI) and reverse primer 5'-GTCGACTAACGCTGCGGCAGAG GC-3' (with SalI). The AvMaSp fragment was inserted into the binary pPZP-3'PINII-Bar vector (pAvMaSp-Bar) containing the seed-specific GluC promoter and the Tnos terminator sequence. To generate transgenic rice containing the partial AvMaSp gene, pAvMaSp-Bar was introduced into Agrobacterium tumefaciens (EHA105) by electroporation. A modified version of a general rice transformation protocol was used (ToKi, 1997; Mohanty et al., 1999). Rice grains were prepared using a rice milling machine and processed to a fine powder using a blender for oral administration. The compositions of both control (Dongjin rice) and transgenic rice powders were the same, including equal proportions of casein, sucrose, corn oil, cellulose, vitamin mixture, mineral mixture, choline bitartrate, DL-methionine, and t-butylhydroquinone.

\section{Animals and sampling}

Diabetic male BKS.Cg-m+/+Leprdb mice (8 weeks of 
age) were purchased from Samtako Bio-Korea Co. (Osan, Korea). The mice were housed in a climate-controlled $\left(21 \pm 2^{\circ} \mathrm{C}\right)$ animal room at a constant 12 -h light/dark cycle. All procedures were performed in accordance with protocols approved by the Dong-A University Animal Care and Use Committee (DIACUC-19-13). Each control or transgenic rice powder was provided in a separate feeding container ad libitum once per day. After 4 weeks of feeding, the mice ( $n=15$ per group) were sacrificed by carbon dioxide asphyxiation and whole blood was collected by cardiac puncture. The abdominal fat samples were finely dissected, weighed, and immediately used for the protein analysis and histological study.

\section{Determination of blood glucose level}

Plasma glucose concentrations were measured in tail blood using a GlucoDr Blood Glucose Test Strip (Hasuco, Seoul, Korea).

\section{Western blot analysis}

Small pieces of abdominal adipose tissues were homogenized in lysis buffer [300 mM NaCl, 0.5\% Triton X-100, $50 \mathrm{mM}$ Tris- $\mathrm{HCl}$ (pH 7.4), $25 \mathrm{mM} \mathrm{NaF}, 1 \mathrm{mM} \mathrm{Na}_{3} \mathrm{VO}_{4}, 10$ $\mathrm{mM} \mathrm{Na} \mathrm{P}_{2} \mathrm{O}_{7}$, and protease inhibitor] for $40 \mathrm{~min}$ on ice. The lysates were centrifuged at $13,000 \times \mathrm{g}$ at $4^{\circ} \mathrm{C}$ for 20 min, the supernatants were collected and protein concentration was measured using the BCA protein assay kit. 30 $\mu \mathrm{g}$ of protein extract with sodium dodecyl sulfate (SDS)loading buffer was electrophoretically separated on a $8 \%-$ $15 \%$ gradient SDS-PAGE gel, and transferred onto a nitrocellulose membrane. The membranes were blocked with $5 \%$ non-fat dry milk dissolved in Tris-buffered saline (TBS) buffer containing $0.05 \%$ Tween-20 at RT for $1 \mathrm{~h}$. The blots were incubated with primary antibodies, followed by incubation with appropriate HRP-conjugated secondary antibodies. The signals were detected with ECL detection reagent in the LAS-4000 (Fuji, Tokyo, Japan). $\beta$-Tubulin was used as internal control for total cellular proteins.

\section{Immunohistochemistry}

Abdominal adipose tissues were fixed in Bouin's solution, embedded in paraffin, and sectioned at $5 \mu \mathrm{m}$ thickness. Tissue sections were placed on glass slides, deparaffinized, hydrated, and treated with $1 \%$ hydrogen peroxide $\left(\mathrm{H}_{2} \mathrm{O}_{2}\right)$ for $10 \mathrm{~min}$ to suppress endogenous peroxidase activity. Antigen retrieval was performed by heating the sections in $1 \mathrm{mM}$ citric acid solution ( $\mathrm{pH}$ 6.0). Sections were incubated with the primary antibody for AMPKa, IRS1, pIRS1 (Tyr632) and STAMP2 at $4^{\circ} \mathrm{C}$ overnight and reacted with biotinylated horse anti-mouse $\mathrm{IgG}$ for $1 \mathrm{~h}$ at $\mathrm{RT}$, as per the manufacturer's instructions for Vectastain Elite ABC kit. The stained sections were developed with liquid diaminobenzidine and counterstained with hematoxylin. The results were observed using a ScanScope digital slide scanning system (Aperio Technologies, Vista, CA, USA).

\section{Statistics}

Data were expressed as the mean \pm SD of at least 3 independent experiments. The difference in means between 2 groups was analyzed using the Student's $t$-test. Mean values were considered significantly different at $p<0.05$.

\section{RESULTS AND DISCUSSION}

In the present study, feeding diabetic mice with TR-SPF caused significant reduction of their blood glucose levels (Fig. 1C) without changes in body weight (Fig. 1A) and abdominal fat mass (Fig. 1B). However, histological observation showed that the adipocyte size in abdominal fat in TR-SFP-fed mice was remarkably smaller than that of the control (Fig. 1D). These results indicated that although the fat mass and weight were not noticeably reduced after TRSPF feeding, subtle cellular and molecular metabolic changes in adipocytes may correlate with lipolysis in adipocytes in these diabetic mice. In T2DM, the potent involvement of adipocytes in balancing glucose metabolism has been implicated together with that of the liver, skeletal 

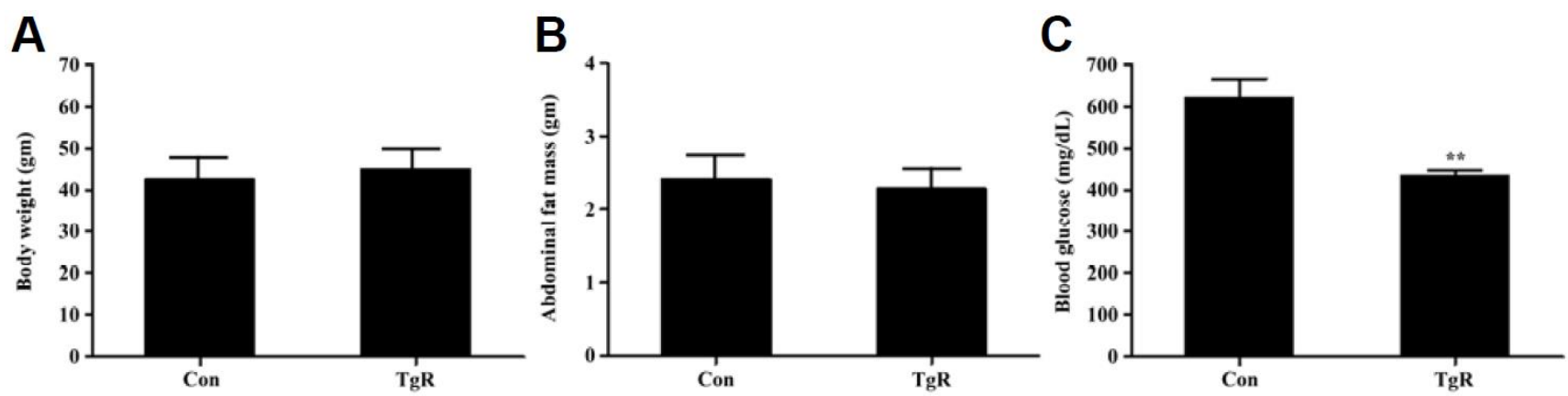

D

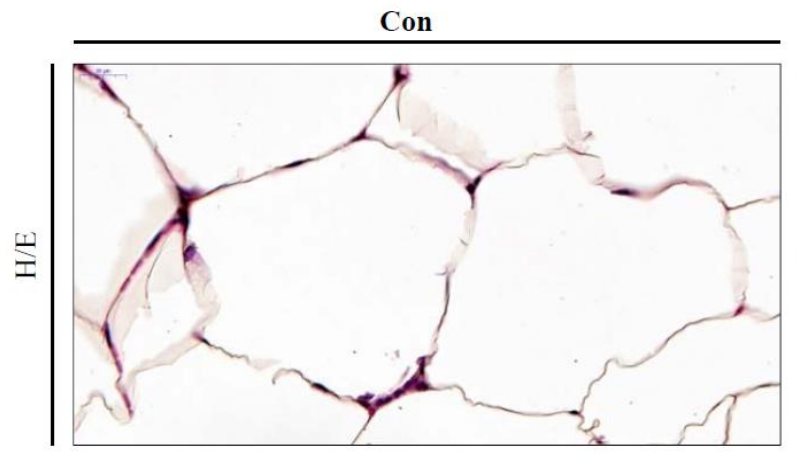

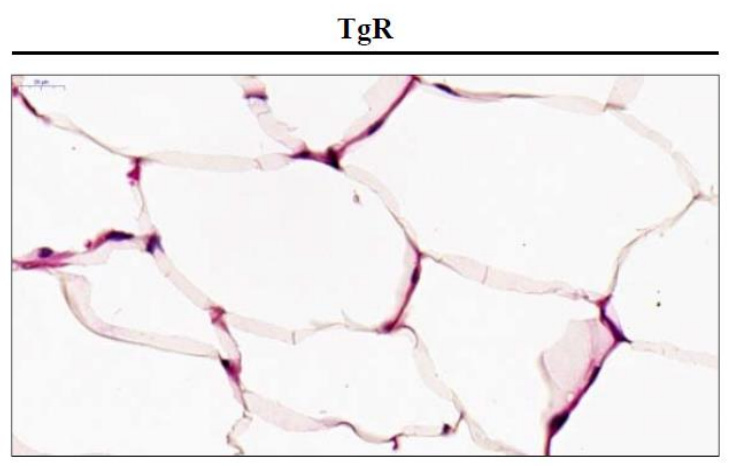

Fig. 1. Changes in body weight (A), abdominal fat mass (B), blood glucose levels (C), and abdominal fat histology (D) after feeding diabetic mice TR-SPF for 4 weeks. The data are expressed as mean \pm SD of three independent experiments performed in triplicates. ${ }^{* *} p<0.01$ compared with those of control (CON). TgR denotes TR-SPF-fed group. (D) Hematoxylin-eosin (H/E) staining. Original magnifications: 800×. TR, transgenic rice; SPF, silk protein fibroin.

muscle, and pancreas (Czech, 2017). Obesity is correlated with the occurrence of T2DM (Boles et al., 2017). Therefore, lipolysis in adipocytes is an important event that modulates IR in T2DM (Morigny et al., 2016). In this context, this study focused on investigating the cellular and biochemical changes in adipocytes during their size reduction after exposure to TR-SPF.

In both hepatocytes and adipocytes, AMPK and IRS1 are known to associate with IR and the pathogenesis of T2DM (Musi \& Goodyear, 2006). Recently, the six-transmembrane protein of prostate 2 (STAMP2) has been also emphasized in the regulation of IR and adipocyte differentiation (Sikkeland \& Saatcioglu, 2013; Kim et al., 2015). In this study, whereas the AMPK and IRS1 protein levels were increased in abdominal adipose tissues after TR-SPF feeding, levels of STAMP2 proteins decreased (Fig. 2A).
In addition, phosphorylation of AMPK at threonine 172 (pAMPK thr172) and IRS1 at serine 307 and tyrosine 632 (pIRS1 ser307/tyr632) were both increased in adipose tissues from TR-SPF-fed mice (Fig. 2A). The changes in these proteins were confirmed in adipose tissues by immunohistochemical localization (Fig. 2B). AMPK is a threonine/ serine kinase that plays an important role in energy metabolism and is a sensor of the cellular energy state that responds to metabolic stresses and other regulatory signals. In hepatocytes, inhibition of AMPK is known to cause IR, whereas activation of AMPK increases insulin sensitivity. Recently, it has been demonstrated that AMPK is activated in adipocytes in which lipolysis is stimulated (Gauthier et al., 2008). Consistent with this, our results also showed that AMPK expression and AMPK phosphorylation were increased in adipose tissues from TR-SPF-fed mice. This 

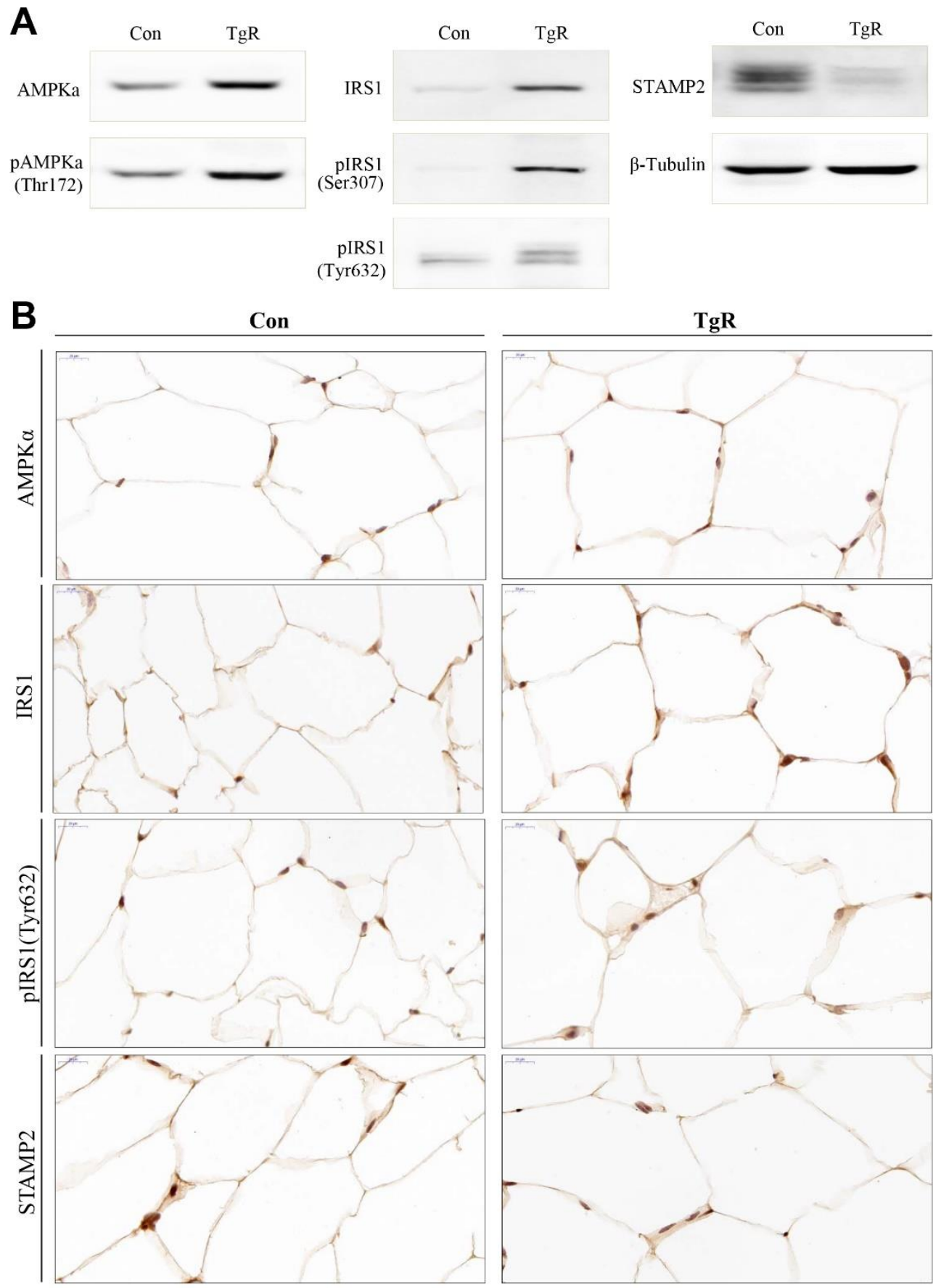

Fig. 2. Alterations in the levels of AMPK, pAMPK (Thr172), IRS1, pIRS1 (Ser307), pIRS1 (Tyr632), and STAMP2 proteins and immunocytochemical localizations of AMPK, IRS1, pIRS1 (Tyr632), and STAMP2 in abdominal fat tissues after feeding diabetic mice TR-SPF for 4 weeks. (A) SDS-PAGE followed by western blotting was performed using $25 \mu \mathrm{g}$ of protein lysates from fat tissues. $\beta$-Tubulin was used as an internal control for all cellular proteins. (B) Abdominal fat tissues were fixed, paraffin-embedded, and immunostained with corresponding antibody, and observed under a light microscope. Original magnification: 800×. AMPK, adenosine monophosphate-activated protein kinase; IRS1, insulin receptor substrate 1; STAMP2, six-transmembrane protein of prostate 2; TR, transgenic rice; SPF, silk protein fibroin. 
indicated that feeding TR-SPF to a diabetic mouse provoked lipolysis in adipocytes through the activation of AMPK and thereby reduced adipocyte size. IRS1 serves as a hub for transmitting the insulin signal. Most of the phosphorylation of IRS1 at serine/threonine residues has been associated with inhibition of insulin signaling. However, phosphorylation of IRS1 at serine/threonine residues has also been shown to enhance the phosphorylation of tyrosine in IRS1 in response to insulin and hence to stimulate insulin signaling (Danielsson et al., 2005). Increased expression and phosphorylation of IRS1 at both serine 307 and tyrosine 632 in adipose tissues in this study indicated that adipocytes obtained from abdominal fat in TR-SPFfed mice were more susceptible to insulin signaling than that of the control. Previously, it has been suggested that STAMP family proteins are associated with adipocyte differentiation (Sikkeland \& Saatcioglu, 2013). Hepatic STAMP2 alleviates high fat diet-induced hepatic steatosis and IR (Kim et al., 2015). In addition, STAMP2 expression is elevated in fed mice compared to fasting mice (Wellen et al., 2007). In the present study, STAMP2 protein levels decreased in adipose tissues from TR-SPF-fed mice, indicating that STAMP2 proteins were reducing adipocytes that were undergoing lipolysis. However, this cellular and molecular event in adipocytes needs to be further elucidated in the near future.

Taken together, this study showed that TR-SPF was effective in reducing blood glucose levels in diabetic mice and that concurrent lipolysis in abdominal adipocytes was associated with alterations of AMPK, IRS1, and STAMP2. Among these, increased IRS1 expression and its phosphorylation by TR-SFP were considered to be particularly important in the induction of lipolysis in adipocytes, as well as in reducing blood glucose levels in this animal model. Finally, the underlying mechanism(s) by which TR-SPF was able to lower blood glucose levels in diabetic mice will become more obvious with further comparative studies in hepatic tissues.

\section{CONFLICT OF INTERESTS}

The authors declare no potential conflict of interest.

\section{ACKNOWLEDGMENTS}

This study was supported by a grant from the NextGeneration BioGreen 21 Program (Project No. PJ01368402), Rural Development Administration, Korea.

\section{REFERENCES}

Altman GH, Diaz F, Jakuba C, Calabro T, Horan RL, Chen J, Lu H, Richmond J, Kaplan DL (2003). Silk-based biomaterials. Biomaterials 24:401-416.

Boles A, Kandimalla R, Reddy PH (2017) Dynamics of diabetes and obesity: Epidemiological perspective. Biochim Biophys Acta Mol Basis Dis 1863:1026-1036.

Czech MP (2017) Insulin action and resistance in obesity and type 2 diabetes. Nat Med 23:804-814.

Danielsson A, Ost A, Nystrom FH, Stralfors P (2005) Attenuation of insulin-stimulated insulin receptor substrate-1 serine 307 phosphorylation in insulin resistance of type 2 diabetes. J Biol Chem 280:34389-34392.

Do SG, Park JH, Nam H, Kim JB, Lee JY, Oh YS, Suh JG (2012) Silk fibroin hydrolysate exerts an anti-diabetic effect by increasing pancreatic $\beta$ cell mass in C57BL/KsJ$\mathrm{db} / \mathrm{db}$ mice. J Vet Sci 13:339-344.

Gauthier MS, Miyoshi H, Souza SC, Cacicedo JM, Saha AK, Greenberg AS, Ruderman NB (2008) AMP-activated protein kinase is activated as a consequence of lipolysis in the adipocyte: potential mechanism and physiological relevance. J Biol Chem 283:16514-16524.

Gotoh K, Izumi H, Kanamoto T, Tamada Y, Nakashima H (2000) Sulfated fibroin, a novel sulfated peptide derived from silk, inhibits human immunodeficiency virus replication in vitro. Biosci Biotechnol Biochem 64:1664-1670. 
Hyun CK, Kim IY, Frost SC (2004) Soluble fibroin enhances insulin sensitivity and glucose metabolism in 3T3-L1 adipocytes. J Nutr 134:3257-3263.

Igarashi K, Yoshioka K, Mizutani K, Miyakoshi M, Murakami T, Akizawa T (2006) Blood pressure-depressing activity of a peptide derived from silkworm fibroin in spontaneously hypertensive rats. Biosci Biotechnol Biochem 70:517-520.

Jung EY, Lee HS, Lee HJ, Kim JM, Lee KW, Suh HJ (2010) Feeding silk protein hydrolysates to C57BL/KsJ$\mathrm{db} / \mathrm{db}$ mice improves blood glucose and lipid profiles. Nutr Res 30:783-790.

Kim HY, Park SY, Lee MH, Rho JH, Oh YJ, Jung HU, Yoo SH, Jeong NY, Lee HJ, Suh S, Seo SY, Cheong J, Jeong JS, Yoo YH (2015) Hepatic STAMP2 alleviates high fat diet-induced hepatic steatosis and insulin resistance. J Hepatol 63:477-485.

Kluge JA, Rabotyagova O, Leisk GG, Kaplan DL (2008) Spider silks and their applications. Trends Biotechnol 26:244-251.

Lee KS, Kim BY, Kim DH, Jin BR (2012) Molecular cloning and characterization of the partial major ampullate silk protein gene from the spider Araneus ventricosus. J Asia Pac Entomol 15:641-646.

Lee KS, Kim BY, Kim DH, Jin BR (2014) Spider silk fibroin enhances insulin secretion and reduces blood glucose levels in diabetic mice. J Asia-Pacific Entomol 17:907-909.

Lee KS, Kim BY, Kim DH, Jin BR (2016) Recombinant spider silk fibroin protein produces a non-cytotoxic and non-inflammatory response. J Asia-Pacific Entomol 19: 1015-1018.

Mohanty A, Sarma NP, Tyagi AK (1999) Agrobacteriummediated high frequency transformation of an elite indica rice variety Pusa Basmati 1 and transmission of the transgenes to R2 progeny. Plant Sci 147:127-137.

Morigny P, Houssier M, Mouisel E, Langin D (2016) Adipocyte lipolysis and insulin resistance. Biochimie 125: 259-266.

Musi N, Goodyear LJ (2006) Insulin resistance and improvements in signal transduction. Endocrine 29:73-80.

Park KJ, Jin HH, Hyun CK (2002) Antigenotoxicity of peptides produced from silk fibroin. Process Biochem 38:411-418.

Rena G, Hardie DG, Pearson ER (2017) The mechanisms of action of metformin. Diabetologia 60:1577-1585.

Sanchez-Rangel E, Inzucchi SE (2017) Metformin: clinical use in type 2 diabetes. Diabetologia 2017 60:1586-1593.

Sikkeland J, Saatcioglu F (2013) Differential expression and function of stamp family proteins in adipocyte differentiation. PLOS ONE 8:e68249.

Thomas CC, Philipson LH (2015) Update on diabetes classification. Med Clin North Am 99:1-16.

Toki S (1997) Rapid and efficient Agrobacterium-mediated transformation in rice. Plant Mol Bio Rep 15:16-21.

Wellen KE, Fucho R, Gregor MF, Furuhashi M, Morgan C, Lindstad T, Vaillancourt E, Gorgun CZ, Saatcioglu F, Hotamisligil GS (2007) Coordinated regulation of nutrient and inflammatory responses by STAMP2 is essential for metabolic homeostasis. Cell 129:537-548. 